angina pectoris or myocardial infarction. A group of the 211 healthy subjects (M/F: 152/59) were the controls. Hyperlipoproteinemia, especially type IIa and type IIb, was more frequent in the CCU patients. We investigated lipoprotein cholesterol levels in the CCU patients and the controls according to 10-year age groupings. LDL-cholesterol levels in the CCU group decreased with age, whereas those in the control group increased. They were higher in male CCU patients under 60 years, and in female patients at all ages, than in controls. The frequency of apo E4 was higher and that of E2 was lower in the CCU group than in the control group. Apo E mutants, E7 and E5, were also frequent in the CCU group. There was no striking difference between the controls and older male patients. These differences were the most obvious in females, followed by younger male patients (Fig. 1). The results suggested that the E4 and E2 isoproteins were positive and negative risk factors for the development of atherosclerosis, respectively. One of the mechanisms of atherosclerosis is associated with the action of apo $\mathrm{E}$ isoproteins on serum LDL levels.

\title{
Supplementary. Significant Role of Apo E in Lipoprotein Metabolism in Rabbits
}

\author{
Nobuhiro YAMADA and Shun ISHIBASHI
}

The Third Department of Internal Medicine, University of Tokyo, Tokyo

Apolipoprotein E (apo E) is known to play an important role in lipoprotein metabolism. We have studied the effect of apo $\mathrm{E}$ on the metabolism of plasma cholesterol by injecting apo E intravenously into rabbits deficient in LDL receptors (WHHL). LDL cholesterol is mainly removed from blood plasma by hepatic LDL receptors that interact with two specific ligands on lipoproteins, apolipoprotein B-100 (apo B-100) and apo E. Lipoproteins rich in apo $\mathrm{E}$, such as large VLDL and $B$ VLDL, have a much higher affinity for LDL receptors than LDL, which contains only apo B-100. In addition, lipoproteins containing apo E can bind to receptors on the liver cell membranes that are responsible for the uptake of dietary cholesterol carried in chylomicron remnants. This chylomicron remnant receptor is thought to be distinct from the LDL receptor because chylomicron remnants are normally removed from the blood in human familial hypercholesterolemia and in WHHL rabbits.

Recent research has demonstrated two lipoprotein subclasses containing apo B-100; B, E particles containing apo $\mathrm{E}$ and $-\mathrm{B}$ and $\mathrm{B}$ particles lacking apo $\mathrm{E}$. The presence of apo $\mathrm{E}$ has been shown to have a profound influence on the removal of apo B-100 in VLDL particles from the blood of normal rabbits and their conversions of higher density (IDL and LDL). In WHHL rabbits, the rate of removal of VLDL-B,E particles from the blood was 4-fold higher than that of VLDL-B particles. These results suggest that in WHHL rabbits a small number of LDL receptors expressed on the surface of hepatocytes or chylomicron remnant receptors participate in the removal of lipoproteins containing several molecules of apo $\mathrm{E}$ by interacting with apo $\mathrm{E}$.

The WHHL rabbit is an excellent animal model to test the effect of agants that could alter the interaction of lipoproteins with lipoprotein receptors. In the present study, we injected massive amounts of apo E into WHHL rabbits to enrich lipoproteins in the receptor-active protein. We speculated that such lipoproteins would be removed much more efficiently through either the chylomicron-remnant receptor or the mutant LDL receptor resulting in the decreased level of plasma cholesterol.

Approximately $30 \mathrm{mg}$ of apo $\mathrm{E}$ was injected per rabbit; a total of five WHHL rabbits were used. One hour later, plasma cholesterol level fell $8.3 \%$ (from $488 \pm 192$ to $446 \pm 174 \mathrm{mg} / \mathrm{dl}$ ). After 3 hours, 
cholesterol levels had fallen by $19 \%$ (to $392 \pm 152$ $\mathrm{mg} / \mathrm{dl}$ ). The reduced levels were maintained for at least 8 hours after injection of apo E. Cholesterol in VLDL and IDL fell rapidly during the first 2 hours after injection, followed by a reduction in the LDL cholesterol level. Changes in apolipoprotein B levels in each lipoprotein fraction were very similar to those of cholesterol. Plasma apo E levels 3 minutes after injection were elevated 3 -fold to $22.8 \pm 6.3$ $\mathrm{mg} / \mathrm{dl}$ and returned initial levels 8 hours after injection. The rate of removal of intravenously injected labelled VLDL that had been incubated with apo E was 3-fold higher than that of unmodified VLDL. From these results, we conclude that the injected apo
$\mathrm{E}$ is incorporated into VLDL and that VLDL particles carrying more apo $\mathrm{E}$ are removed from the blood more rapidly, resulting in reduced formation of LDL and lowered plasma cholesterol levels.

Further research, including studies of the effects of sustained administration of apo E, is needed to determine the fate of cholesterol leaving the blood. after injection of apo E. Apo E has been producéd biosynthetically by a bioengineering technique, and its availability should make it possible to determine whether apo $\mathrm{E}$ can be used to reduce the rate of atherogenesis in WHHL rabbits or other suitable animal models of accelerated atherogenesis.

\title{
4. Treatment of Refractory Hyperlipidemia
}

\author{
Yasushi SAITO, Masaki SHINOMIYA, Kohji SHIRAI and Sho YOSHIDA
}

The Second Department of Internal Medicine, School of Medicine, Chiba University, Chiba

Hypolipidemic drugs developed recently can lower the plasma lipid level more than those of the previously used ones. The final goal to treat the hyperlipidemics is to prevent or improve complication due to hyperlipidemia. Even if the plasma lipid level is the same, the response to hypolipidemic drugs or the contribution of hyperlipidemia to the development of atherosclerosis, is not usually constant. To overcome these problems, we must take it into consideration that the effects are different in each individuals. Considering these problems mentioned above, we will describe the following three themes; (I) The effect of medium chain triglyceride (MCT) on type V hyperlipidemia, (II) The effect of recent therapies for the hypercholesterolemia, (III) the drug effects on plasma triglyceride or HDL-cholesterol levels.

\section{(I) The effect of MCT on type $V$ hyperlipidemia}

The emergent therapy of hypertriglyceridemia was the fasting or reduction of fat intake. Although these are very effective, the treatment of recurrent pancreatitis due to hypertriglyceridemia is not easy, because severe restriction of calory intake could not be continued for long time. The cause of type I or $\mathrm{V}$ hyperlipidemia has been considered to be the deficiency of lipoprotein lipase $(\mathrm{LpL})$ or apo-CII. We found the new type of $\mathrm{LpL}$ abnormality in type $\mathrm{V}$ hyperlipidemia. Patient was 14 years old female suffering from the recurrent abdominal pain due to pancreatitis. LpL activity was not detected in patient' post-heparin plasma. However, the reactive protein to the monoclonal antibody against $\mathrm{LpL}$ was detected, suggesting the existence of $\mathrm{LpL}$ protein molecule. Therefore, we diagnosed this patient as LpL-function deficiency. This defective $\mathrm{LpL}$ could not hydrolyze emulsified triolein in vitro, but could hydrolyze triolein when the triolein was emulsified with tricaprin. Therefore, we administered MCT containing diet to the patient. Patient plasma triglyceride and amylase level was remarkably decreased and abdominal pain subsided. 